\title{
Recent advances in toxicological testing of the stratum corneum
}

\author{
G.E. Piérard, C. Piérard-Franchimont, T. Hermanns-Lê and P. Paquet \\ Laboratory of Skin Bioengineering and Imaging, Department of Clinical Sciences, University of Liège, Liège, Belgium
}

\section{Summary}

\section{Correspondence}

Gérald E. Piérard.

E-mail: gerald.pierard@ulg.ac.be

\section{Accepted for publication}

29 May 2014

\section{Funding sources \\ None.}

\section{Conflicts of interest \\ None declared.}

DOI $10.1111 /$ bjd.13244
$\boldsymbol{\alpha}$-Hydroxy acid (AHA) formulations are commonly used for skin chemical peelings. The primary target is the stratum corneum (SC). The aim of this study was to assess the effects of various glycolic acid concentrations and commercial phenolic acid formulations on the SC. Quantitative colorimetry of a corneoxenometry bioassay was used. The test procedure involved glycolic acid concentrations ranging from 3\% to $70 \%$ in alcoholic solution. Exposure times were set for $1 \mathrm{~min}$ and $3 \mathrm{~min}$. The bioassay showed consistent reactivity with a dose-effect relationship when using the selected low exposure times. In a similar procedure the aggressiveness of commercially available phenolic acid formulations was identified not using hazardous in vivo testing. Corneoxenometry appears useful for in vitro testing of AHA peeling agents during short exposure times.

\section{What's already known about this topic?}

- Alpha hydroxyacids (AHA) are used for chemical peelings.

- AHA dosages used in clinical practice clearly depend on the chemical nature of the chemical compound.

- There is no safe clinical predictive test determining the safety of AHA concentrations and formulations.

\section{What does this study add?}

- Corneoxenometry (CXM) is offered as an ex-vivo bioassay predicting interactions between AHA and the human stratum corneum.

- Other xenobiotics distinct from AHA and possibly altering corneocytes could be tested using CXM in non hazardous procedures.
Glycolic acid (GA) and phenolic acid (PA) are $\boldsymbol{\alpha}$-hydroxy acids (AHAs) used for improving the severity of skin roughness, xerosis, retentional acne, some melanoses and several signs of photoageing. ${ }^{1-6}$ The complete mechanisms of action of GA on the stratum corneum (SC) are yet to be completely identified. Enhanced corneodesmosome breakdown following topical applications of low GA concentrations was observed using electron microscopy. ${ }^{7}$ Such a cleavage process is possibly related to the activity of intercorneocyte cathepsin D-like proteinases. ${ }^{8}$ In addition, various AHAs appear to stimulate ceramide biosynthesis, ${ }^{9}$ in turn thwarting any faulty desquamation process, and increasing SC moisture levels. ${ }^{10}$ When 35-70\% GA concentrations are applied to human skin, a swift decrease in corneocyte cohesion takes place and the stratum Malpighii becomes necrotic. The combination of these effects is clinically observed as fragmentation of the SC into sheet-like fragments. ${ }^{8}$ Long-term effects include a quicker SC turnover, which is responsible for SC thinning. Globally, GA acts as a chemical peeling agent. ${ }^{11,12}$

Comparative clinical assessments of the risk-benefit ratio of different AHA concentrations is difficult to perform. Indeed, testing the effects of chemical peels is potentially hazardous in vivo. By contrast, in vitro or ex vivo bioassays would be more convenient for predicting some AHA-specific effects occurring in clinical practice. ${ }^{13}$

In the present study, the effects of different GA concentrations and PA commercial formulations were tested on normal human SC using the previously described corneoxenometry bioassay (CXM after corneocyte, xenobiotic, metry). ${ }^{13,14}$ This method entails the collection of human SC as a substrate for 
testing the ex vivo reactivity of xenobiotics within the corneocyte layers. The test compound at a given concentration is placed in contact with the SC for a defined period of time. After rinsing the SC with tap water, specific dyes are applied to the samples. The staining intensity is proportional to the degradation of proteins and lipids on the corneocytes. The average colour of the samples measured by reflectance colorimetry is a severity indicator of the damage induced by the xenobiotic to the SC.

\section{Materials and methods}

\section{Experiment 1}

GA concentrations of 3, 5, 7, 10, 20, 30, 50 and 70\% in alcohol were tested at room temperature using CXM. Two series of nine cyanoacrylate skin-surface strippings (CSSSs) were harvested from the volar aspect of both forearms in 30 healthy adults. One CSSS from each volunteer was dipped for 1 or 3 min in water or in one of each of the GA test solutions. After rinsing under running tap water, they were air dried and stained for $30 \mathrm{~s}$ in a $30 \%$ hydroalcoholic solution of toluidine blue and basic fuchsin. Each CSSS colour was measured in triplicate by reflectance colorimetry using a Chroma Meter ${ }^{\circledR}$ CR400 (Konica-Minolta, Osaka, Japan). The mean CXM colorimetric index of mildness (CIM), representing the staining intensity of the samples, was calculated as previously reported, ${ }^{13,14}$ as follows: $\mathrm{CIM}=$ $\mathrm{L}^{*}$-Chroma $\mathrm{C}^{*}$. The CIM value decreases with increasing chemical alteration of the corneocyte envelope.

CIM data were reported as means and SDs. Comparisons between series of data were made using the Student's paired t-test. A P-value $<0.05$ was considered statistically significant.

\section{Experiment 2}

Commercially available peeling formulations containing PA were prepared at the reported $4.4 \%$ and $10 \%$ concentrations. The first patient who received the treatment experienced severe chemical burns on the face. Three products - A, B and C - had been sequentially applied, and CXM was applied to the three formulations used singly and sequentially. The procedure was similar to the short CXM design described for GA, but lasted only $30 \mathrm{~s}$ for the PA formulations. In addition, gas chromatography was performed in a second step for dosing the actual PA concentrations in the three formulations.

\section{Results}

\section{Experiment 1}

CIM data by GA concentration are presented in Figure 1. The CIM values obtained after a 1-min exposure time progressively decreased with increasing GA concentrations. Significance ( $P<0.05)$ was reached from the $20 \%$ concentration onwards. No statistically significant differences were seen between any successive levels of the tested GA concentrations.

CIM values for the 3-min GA exposure progressively decreased with increasing GA concentration to reach significance $(\mathrm{P}<0.05)$ at $10 \%$ concentration (Fig. 1). Additional increase in GA concentration led to a greater decrease in CIM values.

The two different GA exposure times ( 1 vs. $3 \mathrm{~min}$ ) yielded significantly different CIM values $(\mathrm{P}<0.05)$ from the $20 \%$ concentration onwards.

\section{Experiment 2}

For two of the tested PA formulations (A, B) at the $10 \%$ and $4.4 \%$ concentrations, respectively, the CIM data were in the expected range, reaching 39.72 and $53 \cdot 42$, respectively. By contrast, formulation $\mathrm{C}$ at the reported $4.4 \%$ concentration showed a reduction of the CIM value to 5.09 (Fig. 2). Similarly, sequential CXM applications of the three products gave a minimal CIM value of $1 \cdot 23$

Gas chromatography revealed a PA concentration in product C about 1000-fold higher than the claimed concentration.

\section{Discussion}

The present study focused on the relevance of CXM for assessing the effects of various AHA concentrations on the SC. CXM resembles corneosurfametry, which is designed specifically for testing surfactants. ${ }^{15,16}$ CIM data yielded by both bioassays

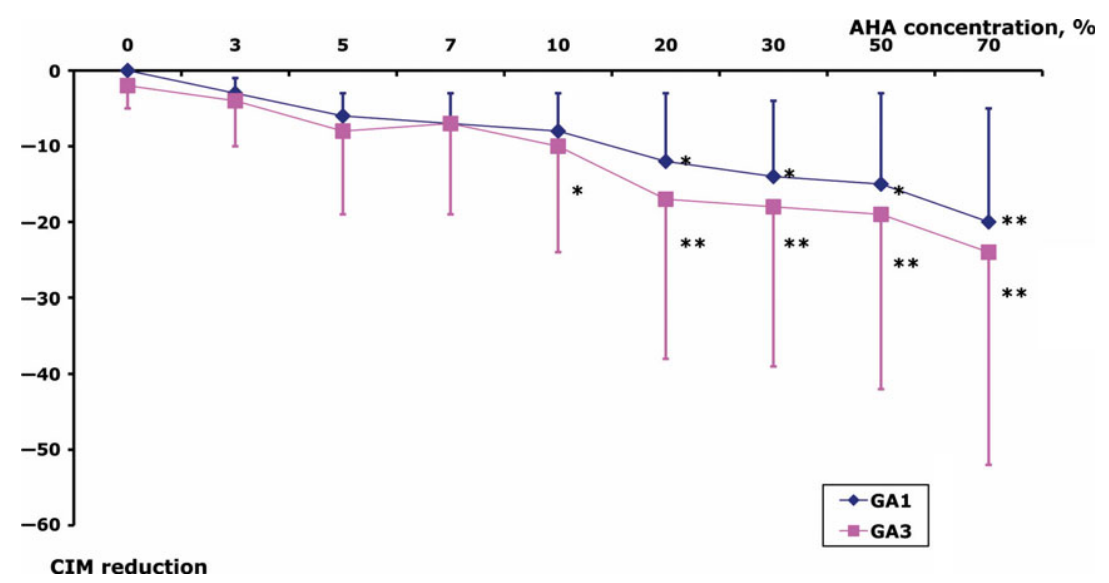

Fig 1. Compared with water as a control, reduction in colorimetric index of mildness (CIM) is influenced by various glycolic acid (GA) concentrations during $1 \mathrm{~min}(\mathrm{GAl})$ and 3 min (GA3) contact time with human stratum corneum. $* \mathrm{P}<0.05, * * \mathrm{P}<0.01$ for comparison between the effects on CIM at each time point. AHA, $\boldsymbol{\alpha}$-hydroxy acid. 


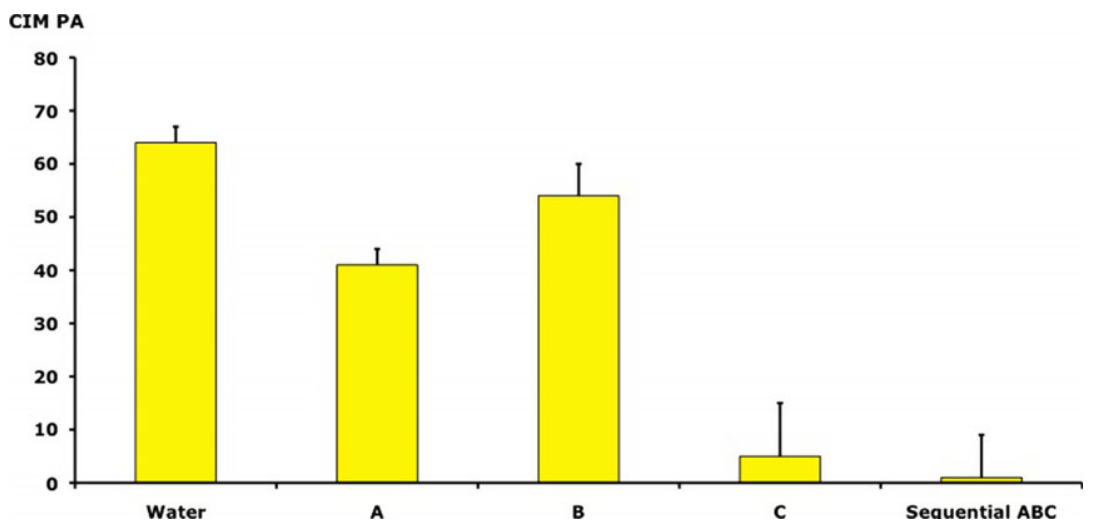

reflect the alterations of the SC induced by the test products. A previous study had shown that both the nature and concentration of the test product, as well as the exposure time, represented important variables influencing the CIM data. ${ }^{13}$ The same bioassay allows the testing of so-called skin protection creams. ${ }^{17}$ A correlation was previously established between corneosurfametry data and predictive testing on human skin. $^{15,18}$

Topically applied GA has proven its relative efficacy and safety in a variety of skin conditions. ${ }^{2,4}$ Histologically, the recognized effects include reduction of SC thickness contrasting with increased epidermal thickness, more orderly maturation, enhanced rete ridge pattern, and dispersal of melanin within the basal layer. ${ }^{17,19}$ Additionally, GA possibly induces changes in the dermis. ${ }^{2}$

The present study shows the reactivity of various GA concentrations in the CXM bioassay. The relationship between CIM and GA concentration indicates that both the 1- and 3-min exposure times were convenient and well suited to the purpose. In contrast, a previous study using a longer exposure had yielded some erratic CIM values in the CXM bioassay. ${ }^{13}$ This recommended short exposure times for testing peeling agents with CXM. This procedure represents a major difference from the 2-h corneosurfametry procedure used for testing mild surfactant-based skin-cleansing products. The short time of the CXM procedure is closer to that required for microwave corneosurfametry. ${ }^{15}$

CXM applied to commercially available PA peeling products rapidly yielded information about their safety and, on this occasion, clearly identified dangerous instructions in one of the formulations.

In conclusion, the effects of two AHAs on human SC were tested ex vivo and at various concentrations. Once the most convenient exposure time is determined for any given AHA to guarantee the sensitivity of the CXM bioassay, the procedure can be safely used to compare the CIM values of various peeling agents alone or in combination, and at various concentrations.

\section{References}

1 Tung RC, Bergfeld WF, Vidimos AT, Remzi BK. $\alpha$-Hydroxyacidbased cosmetic procedures. Guidelines for patient management. Am J Clin Dermatol 2000; 1:81-8.
Fig 2. Colorimetric index of mildness (CIM) values of phenolic acid (PA) formulations, indicating a dramatic effect for both product $\mathrm{C}$ and sequential use of $\mathrm{A}, \mathrm{B}$ and $\mathrm{C}$.
2 Funasaka Y, Sato H, Usuki A et al. The efficacy of glycolic acid for treating wrinkles: analysis using newly developed facial imaging systems equipped with fluorescent illumination. J Dermatol Sci 2001; 27 (Suppl. 1):S53-9.

3 Xhauflaire-Uhoda E, Marcq V, Piérard-Franchimont C, Piérard GE. How I treat ... evanescent youth. Dating back skin using chemical peels. Rev Med Liège 2005; 60:761-6.

4 Chaudhary S, Dayal S. Efficacy of combination of glycolic acid peeling with topical regimen in treatment of melasma. J Drugs Dermatol 2013; 12:1149-53.

5 Hassan KM, Benedetto AV. Facial skin rejuvenation: ablative laser resurfacing, chemical peels, or photodynamic therapy? Facts and controversies Clin Dermatol 2013; 31:737-40.

6 Vavouli C, Katsambas A, Gregoriou S et al. Chemical peeling with trichloroacetic acid and lactic acid for infraorbital dark circles. J Cosmet Dermatol 2013; 12:204-9.

7 Fartasch M, Teal J, Menon GK. Mode of action of glycolic acid on human stratum corneum: ultrastructural and functional evaluation of the epidermal barrier. Arch Dermatol Res 1997; 289:404-9.

8 Horiskoshi T, Matsumoto M, Usuki A et al. Effects of glycolic acid on desquamation-regulating proteinases in human stratum corneum. Exp Dermatol 2005; 14:34-40.

9 Rabionet M, Gorgas K, Sandhoff R. Ceramide synthesis in the epidermis. Biochim Biophys Acta 2014; 1841:422-34.

10 Effendy I, Kwangsukstith C, Lee JY et al. Functional changes in human stratum corneum induced by topical glycolic acid: comparison with all-trans retinoic acid. Acta Derm Venereol 1995; $75: 455-8$.

11 Fulton JE Jr, Porumb S. Chemical peels. Their place within the range of resurfacing techniques. Am J Clin Dermatol 2004; 5:17987.

12 Uhoda E, Piérard-Franchimont C, Petit L, Piérard GE. Hydroxyacids. In: Cosmeceuticals. Drugs Versus Cosmetics (Elsner P, Maibach HI, eds), 2nd edn. New York: Marcel Dekker, 2005; 207-18.

13 Xhauflaire-Uhoda E, Piérard-Franchimont C, Piérard GE. Effects of various concentrations of glycolic acid at the corneoxenometry and collaxenometry bioassays. J Cosmet Dermatol 2008; 7:194-8.

14 Piérard-Franchimont C, Henry C, Uhoda E et al. Penetration enhancer assessment by corneoxenometry. In: Percutaneous Penetration Enhancers (Smith EW, Maibach HI, eds). Boca Raton: CRC Press, 2005; 193-298.

15 Goffin V, Piérard GE. Microwave corneosurfametry and the shortduration dansyl chloride extraction test for rating concentrated irritant surfactants. Dermatology 2001; 202:46-8.

16 Xhauflaire-Uhoda E, Loussouarn G, Haubrechts C et al. Skin capacitance imaging and corneosurfametry. A comparative assessment of the impact of surfactants on stratum corneum. Contact Dermatitis 2006; 54:249-53. 
Recent advances in toxicological testing of the stratum corneum, G.E. Piérard et al. $\mathbf{3 7}$

17 Xhauflaire-Uhoda E, Macarenko E, Denooz R et al. Skin protection creams in medical settings: successful or evil? J Occup Med Toxicol 2008; 3:15.

18 Piérard GE, Kligman AM, Stoudemayer T, Lévêque JL. Comparative effects of retinoic acid, glycolic acid and a lipophilic derivative of salicylic acid on photodamaged epidermis. Dermatology 1999; 199:50-3.

19 El Samahy MH, Ghoz MM, Ramzy N. Morphological investigation of chemical peel on photodamaged facial skin. Int J Cosmet Sci 1998; 20:269-82. 